\title{
A Specific Pattern in the Basal Body Temperature Chart during the First Week of Pregnancy May Warn of a Miscarriage Crisis
}

\author{
Mayumi Watanabe ${ }^{1 *}$, Yoshinobu Nakamura2\#, Chikako Tomiyama ${ }^{3}$, Toru Abo \\ ${ }^{1}$ Department of Medical Informatics, Niigata University Medical and Dental Hospital, Niigata, Japan \\ ${ }^{2}$ Nakamura Seikotsuin, Niigata, Japan \\ ${ }^{3}$ Department of Medical Technology, Graduate School of Health Sciences, Niigata University, Niigata, Japan \\ ${ }^{4}$ Toru Abo Research Centre, Niigata, Japan \\ Email: *watanabem62@gmail.com
}

Received 17 March 2016; accepted 24 May 2016; published 27 May 2016

Copyright (C) 2016 by authors and Scientific Research Publishing Inc.

This work is licensed under the Creative Commons Attribution International License (CC BY).

http://creativecommons.org/licenses/by/4.0/

(c) (†) Open Access

\begin{abstract}
We studied the efficiency of acupuncture for patients who were unable to give birth though they had experienced conventional infertility treatment for more than 2 years by medical specialists. We then investigated characteristics of difficult pregnancy cases with measurement of body temperature and observation of basal body temperature (BBT) charts. Thirty-three patients had acupuncture every week for more than 2 years and 17 of them delivered. At the same time, their cutaneous body temperature was measured at five locations. Moreover, patients and acupuncturists had a weekly discussion of the patient's BBT chart. The delivery group showed more balanced and higher cutaneous body temperatures than the miscarriage and no pregnancy groups. The delivery group showed a smooth increase in the BBT chart while the miscarriage group showed a specific pattern in shifting temperatures from low to high zones. Acupuncture might suppress excess sympathetic dominance, which induced hyperthermia as well as interruption of pregnancy. Additionally, weekly discussion and medical advice could help patients. However, further study of males, healthy women, or larger, comparative studies exploring the social and cultural background of other countries were needed to add support to these factors.
\end{abstract}

\section{Keywords}

Basal Body Temperature (BBT) Chart, Miscarriage, Kyusaku Ogino, Acupuncture, Medical Advice

\footnotetext{
"MW and YN contributed equally to this work.

"Corresponding author.
}

How to cite this paper: Watanabe, M., Nakamura, Y., Tomiyama, C. and Abo, T. (2016) A Specific Pattern in the Basal Body Temperature Chart during the First Week of Pregnancy May Warn of a Miscarriage Crisis. Health, 8, 723-729. 


\section{Introduction}

Dr. Kyusaku Ogino, an honorary citizen of the city of Niigata, published his discovery of the date of ovulation in the February issue of the Hokuetsu Medical Journal in 1923. He used his discovery to help infertile women to time intercourse to achieve pregnancy. In 1926, Dr. Theodor Hendrik van de Velde noted that the temperature rise in the latter part of the female cycle was because of the activity of the corpus luteum, the follicle from which the ovum had been released [1]. Their studies and methods paved the way for modern reproductive treatment and natural family planning, which were still widely practiced; many women tracked their basal body temperature (BBT) each day. Thus, body temperature was an important indicator for reproduction.

Currently, fertility in developed countries was declining. For example, the total fertility rate in 2011 was 1.39 in Japan given the rise in the proportion of single individuals, age at marriage, and age at child bearing [2]. Moreover, the Lehman Brothers crashed that triggered the 2008 financial crisis provided a stressful working lifestyle. Stress has a significant effect on body temperature because it often induces sympathetic nerve (SN) dominance inducing hypothermia [3] [4], and stress-induced hypothermia may be another reason for low fertility. Additionally, many women suffer from subjective sensitivity to cold temperatures [5] [6]. The World Health Organization and researchers indicate the usefulness of acupuncture for fertility via modulating autonomic nervous balance [7]-[11]. We also report that acupuncture can suppress excess SN dominance [12].

The purposes of this study were 1) to examine the efficiency of acupuncture for patients who were unable to give birth though they had experienced conventional infertility treatment more than 2 years from medical specialists; and 2) to investigate characteristics of difficult pregnancy cases with measurement of body temperature and observation of BBT charts.

\section{Methods}

\subsection{Patients}

Thirty-three patients, aged 24 to 43 years (average $32.4 \pm 4.9$ years) when they started to have weekly acupuncture, were included in the study. Patients came to Nakamura Seikotsuin (Niigata, Japan) or Yushima Shimizuzaka Clinic (Tokyo, Japan) continuously for more than 2 years between January 2010 and December 2015. Written informed consent was obtained from all subjects and the study was approved by the Medical Ethics Committee of Yushima Shimizuzaka Clinic. All patients had experienced conventional infertility treatment more than 2 years from medical specialists; however, they had not given birth in the past. Patients were divided into 3study groups: the delivery group ( $n=17$; age, 26 - 41 years; average age $32.5 \pm 4.7$ years), the miscarriage group ( $n=5$; age, $30-42$ years; average age $34.4 \pm 4.6$ years) and the no-pregnancy group $(n=11$; age, $24-43$ years; average age $33.1 \pm 5.5$ years) (Figure 1 ). The delivery group became pregnant after they had acupuncture treatment for $24 \pm 10$ months (average). However, other groups did not get pregnant though they came to clinic 2 years without interruption in the treatment period.

\subsection{Acupuncture (Dizhen) Application}

An ancient type of acupuncture needle, Dizhen (DZ), which was made of stainless steel (Soken Medical Co. Ltd., Tokyo, Japan), was used for acupuncture treatment. As we consider DZ application advantageous and safe in modern clinical practice, it can avoid the issues with infection and tissue damage sometimes seen with modern

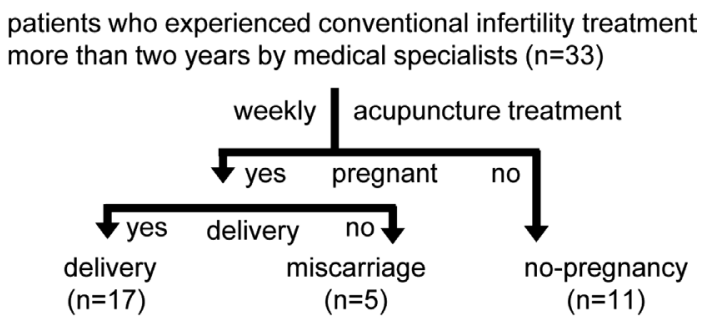

Figure 1. Overview of the 33 patients and 3 study groups: a delivery group, a miscarriage group and a no-pregnancy group. 
acupuncture needles [12] [13].

An autoclaved DZ was softly pressed vertically onto the body surface for 5 minutes (diameter $5.0 \mathrm{~mm}, 37.3$ $\mathrm{g} / \mathrm{mm}^{2}$ ), at approximately 120 points from the head to the feet on both sides of the spine as reported previously [12] [13]. The acupuncture points corresponded to the Conception Vessel, Governor Vessel and the Bladder Meridian as defined by the World Health Organization [14]. Treatment was applied by nationally licensed experienced Japanese acupuncturists. The application and experiments were conducted at a constant temperature of $25.0^{\circ} \mathrm{C} \pm 0.5^{\circ} \mathrm{C}$ and humidity of $50.0 \% \pm 5.0 \%$.

\subsection{Body Temperature Measurement at Five Locations Every Time before Acupuncture Application}

Each time a patient received acupuncture, her cutaneous body temperatures were measured (before and after acupuncture) using a contactless digital thermometer (Microlife, IR 1DA1, Microlife, Windnau, Switzerland) at five previously described peripheral locations: Yintang (on the forehead, midway between the medial ends of the eyebrows), LI4 (Hegu; on the dorsum of the right and left hands, radial to the mid-point of the second metacarpal bone), and LR3 (Taichong; on the dorsum of the right and left feet, between the first and second metatarsal bones, in the depression distal to the junction of the bases of the two bones, over the dorsalis pedis artery) [14].

\subsection{Basal Body Temperature Chart}

We explained to participants the reasons for and importance of tracking BBT under the tongue with basal thermometers each morning, emphasizing a time when they first woke up in the morning after at least 3 hours of uninterrupted sleep. Moreover, we encouraged participants to record a temperature-plotting chart and to bring it with them when they had an acupuncture treatment.

\subsection{Statistical Analysis}

The Mann-Whitney test was conducted and the level of significance was set at $p<0.05$. The values presented are mean \pm S.D. All statistical analyses were performed using SPSS version 20 for Windows (IBM Corp, Armonk, New York, USA).

\section{Results}

\subsection{The Miscarriage Group Showed Balanced and Lower Cutaneous Body Temperatures than the Delivery Group}

Cutaneous body temperatures were measured each time before acupuncture. We compared cutaneous body temperatures of two points; before and the nearest time just before the fertilization in the delivery group (or 2 years later in the miscarriage/no-pregnancy groups). It has been reported that rapid changes in core temperature are associated with changes in cutaneous blood flow mediated by the autonomic nervous system [15]; therefore, we measured cutaneous body temperature over time at five locations.

Weekly acupuncture regulated the shape of pentagon-shaped radar charts (Figure 2). Before acupuncture treatment most patients showed unbalanced and low cutaneous body temperatures in the hands and the legs $\left(<37^{\circ} \mathrm{C}\right)$. After acupuncture treatment, the delivery group tended to show an increase of cutaneous temperature; however, statistical differences were not obtained among 3 groups.

\subsection{Delivery Group Showed a Smooth Increase of Basal Body Temperature Charts}

We made a detailed observation comparing the 3 groups. And then, we extracted BBT records of miscarriage patients $(\mathrm{n}=5)$ and compared with those of other groups (the delivery and no-pregnancy groups) (Figure 3). The miscarriage and no-pregnancy groups did not reach $37^{\circ} \mathrm{C}$ in the week after ovulation (Figure 3(f)-(o)). All patients in the miscarriage group showed a drop in body temperature during the first week after the date of ovulation. The delivery group BBT was elevated in the first week without drooping $\left(>0.15^{\circ} \mathrm{C}\right)$ and reached $37^{\circ} \mathrm{C}$ (Figure 3(a)-(e)). Thus, the delivery group showed a rapid and smooth increase of the BBT chart. In other words, the 2 no-delivery groups showed hyperthermia and miscarriage group indicated slow and dips in increase of BBT chart. 


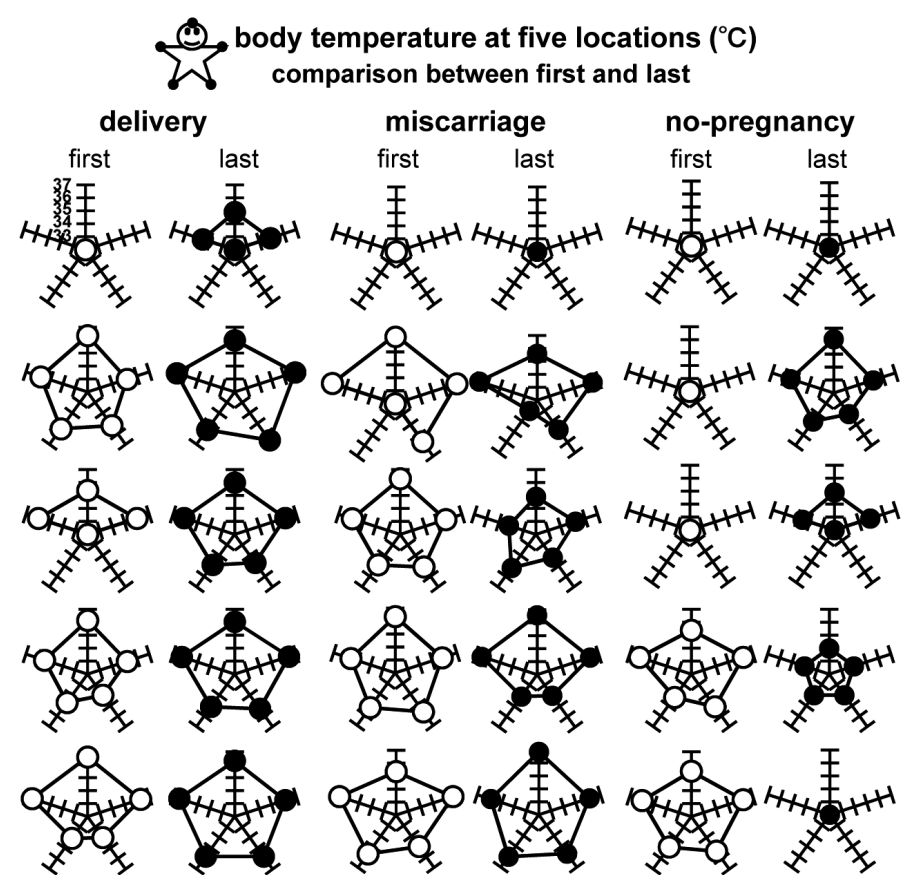

Figure 2. Representative results of delivery and no-pregnancy groups as well as all results of miscarriage group and were depicted. The no-delivery patients (miscarriage and no-pregnancy groups) showed unbalanced and lower cutaneous body temperatures compared with the delivery group. After acupuncture treatment, the delivery group tended to show an increase of cutaneous temperature; however, statistical differences were not obtained among 3 groups.

\section{Discussion}

\subsection{Excess Sympathetic Dominance Induces Hyperthermia as Well as Interruption of Pregnancy}

Our observation revealed two results. First, the miscarriage group showed unbalanced, lower cutaneous body temperatures than the other group. We have reported that low body temperature (hypothermia) is shown under SN dominance because of alpha-adrenergic stimulation, which constricts peripheral blood vessels via alphaadrenergic receptors [3]. Minagawa et al. reported that during pregnancy, women are in a special state of SN dominance and if the activation of sympathetic nerves is too strong during pregnancy, granulocytes and NK cells are activated beyond a certain threshold to interrupt pregnancy [16]. However, acupuncture can induce modest autonomic nervous balance [17]. We hypothesize that acupuncture suppresses SN dominance function (hypothermia) induced by stress; therefore, acupuncture may be beneficial for pregnancy/delivery via modulation of body temperature.

\subsection{The Miscarriage Group Showed a Specific Pattern in Shifting Temperatures from Low to High}

Second, we discovered that the miscarriage group showed a specific pattern in shifting from lower temperatures before ovulation to higher temperatures after ovulation (Figure 3(f)-(j)). It is noteworthy that all patients in the miscarriage group showed a drop in the first week after the date of ovulation. The first week is a very important time for implantation of a fertilized ovum.

Unlike other pituitary and target organ systems, the hypothalamic-pituitary-ovarian system involves multiple trophic hormones with different biological actions (e.g., follicle stimulating hormone and luteinizing hormone) and multiple target hormones having nonlinear feedback functions [18]. Moreover, temperature fluctuates during the normal menstrual cycle as natural hormone levels rise and fall; progesterone nudges the temperature up 


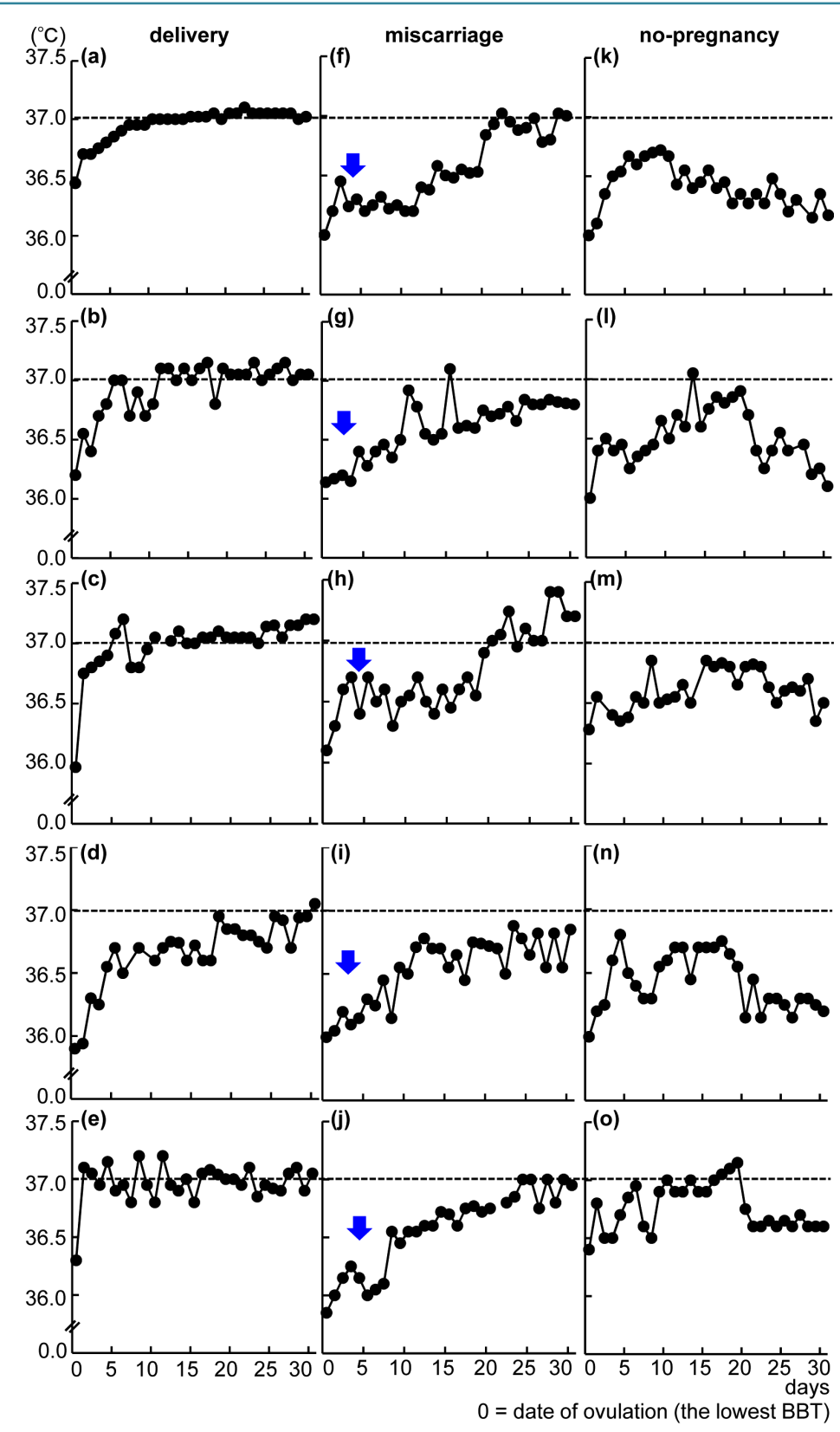

Figure 3. Comparison of the three groups in basic body temperature charts. The delivery group showed a smooth increase in the basal body temperature chart (a)-(e). However, all patients in the miscarriage group showed a temperature drop in the first week after the date of ovulation (f)-(j). The no-pregnancy group showed hyperthermia (k)-(o).

and estrogen brings it down [19]. Such a complicated background exists behind implantation of a fertilized ovum. Continuous stress and induction of SN dominance could affect the fate of fertilized ova because stress related hormones (e.g., acetylcholine) are released from the anterior pituitary gland [20]. In addition to gonadotropins (luteinizing hormone and follicle stimulating hormone), progesterone is regulated via the hypothalamicpituitary-ovarian system [18].

\subsection{Careful Observation of BBT Chart Can Help Patients Improve Self-Management}

A sharp elevation in the BBT chart after ovulation means progesterone has been released, preparing the lining of 
the uterus for the implantation of a fertilized ovum. Therefore, hypothermia and slow shifts or dips in the BBT after ovulation may indicate deficiency of progesterone release, as well as difficulty in maintenance of pregnancy. This is understood to be a reason for miscarriage and no-pregnancy.

Recently researchers reported the fate of fertilized ova (human embryos) with significant genetic abnormalities can alter chemical signals (e.g., trypsin) to produce a stress response in the womb [21]. However, our discovery of the BBT observation, which is simple, easy and practical, is helpful for patients and clinicians. Moreover, BBT observation with patients can help them to improve self-management.

In this way, our findings indicate the efficiency of acupuncture treatment on delivery/pregnancy via suppressing SN dominance and propose a specific sign of miscarriage in this study. However, our study has several limitations. First, we study only patients who have gone through conventional infertility treatment for more than two years. Therefore, we study neither healthy patients nor patients who do not present BBT. Second, half of the outcomes related to delivery and pregnancy depend on the male; however, we do not study men. Third, larger, comparative studies exploring the social and cultural background of other countries are needed to add support to these factors with appropriate statistical analysis.

\section{Acknowledgements}

Special thanks to Mr. Taiki Hashimoto and Ms. Kaori Yamamoto (Yushima-Shimizuzaka Clinic) for arrangement of the clinical research. The authors also thank all of patients who participated in this study.

\section{References}

[1] Kippley, J.F. (2008) Sexual Revolution Part II. Catholic Social Science Review, 13, 373-386. http://dx.doi.org/10.5840/cssr20081332

[2] Kaku, T., Kitahara, E., Murata, S., Noguchi, Y., Shinkoda, H. and Hirata, N. (2001) Problems of Very Low Fertility in Japan. Memoirs of Kyushu University School of Health Sciences, 28, 7-12.

[3] Watanabe, M., Tomiyama-Miyaji, C., Kainuma, E., Inoue, M., Kuwano, Y., Ren, H., Shen, J. and Abo, T. (2008) Role of Alpha-Adrenergic Stimulus in Stress-Induced Modulation of Body Temperature, Blood Glucose and Innate Immunity. Immunology Letters, 115, 43-49. http://dx.doi.org/10.1016/j.imlet.2007.09.010

[4] Kainuma, E., Watanabe, M., Tomiyama-Miyaji, C., Inoue, M., Kuwano, Y., Ren, H. and Abo, T. (2009) Association of Glucocorticoid with Stress-Induced Modulation of Body Temperature, Blood Glucose and Innate Immunity. Psychoneuroendocrinology, 34, 1459-1468. http://dx.doi.org/10.1016/j.psyneuen.2009.04.021

[5] Kawagoe, H., Takahashi, K., Kawashima, A. and Ishikawa, T. (2003) Investigation into the Actual Conditions of Hiesho-The Basic Data and Frequency of Hiesho According to Diseases. Diagnosis and Treatment, 91, 139-142. (In Japanese).

[6] Mori, H., Sakaguchi, S. and Nishijo, K. (2006) Thermographic Guideline of Hiesho. Biomed Thermol, 26, 48-51.

[7] World Health Organization (2003) Acupuncture: Review and Analysis of Reports on Controlled Clinical Trials. World Health Organization, 87.

[8] Manheimer, E., Zhang, G., Udoff, L., Haramati, A., Langenberg, P., Berman, B. and Bouter, L.M. (2008) Effects of Acupuncture on Rates of Pregnancy and Live Birth among Women Undergoing in Vitro Fertilisation: Systematic Review and Meta-Analysis. BMJ, 336, 545-549.

[9] Boivin, J., Griffiths, E. and Venetis, C.A. (2011) Emotional Distress in Infertile Women and Failure of Assisted Reproductive Technologies: Meta-Analysis of Prospective Psychosocial Studies. BMJ, 342, d223. http://dx.doi.org/10.1136/bmj.d223

[10] Louis, G.M., Lum, K.J., Sundaram, R., Chen, Z., Kim, S. and Lynch, C.D., Schisterman, E. and Pyper, C. (2011) Stress Reduces Conception Probabilities across the Fertile Window: Evidence in Support of Relaxation. Fertility and Sterility, 95, 2184-2189. http://dx.doi.org/10.1016/j.fertnstert.2010.06.078

[11] Akbarzadeh, M., Masoudi, Z., Zare, N. and Vaziri, F. (2015) Comparison of the Effects of Doula Supportive Care and Acupressure at the BL32 Point on the Mother's Anxiety Level and Delivery Outcome. Iranian Journal of Nursing and Midwifery Research, 20, 239-246.

[12] Watanabe, M., Takano, O., Tomiyama, C., Matsumoto, H., Urahigashi, N., Kainuma, E., Madarame, T., Fukuda, M. and Abo, T. (2012) The Effects of Acupuncture of an Ancient Type of Acupuncture Needle on Body Temperature, Immune Function and the Autonomic Nerve System. Health, 4, 775-780. http://dx.doi.org/10.4236/health.2012.410120

[13] Watanabe, M., Takano, O., Tomiyama, C., Guan, J., Hou, G., Mori, H., Nishijo, K., Abo, T. and Akazawa, K. (2013) The Effects of Application of an Ancient Type of Acupuncture Needle on Increase in Urination of Hospitalized Old- 
est-Old People. Health, 5, 1092-1098. http://dx.doi.org/10.4236/health.2013.57147

[14] World Health Organization (2002) Acupuncture: Review and Analysis of Reports on Controlled Clinical Trials. World Health Organization, Geneva.

[15] Baker, M.A., Cronin, M.J. and Mountjoy, D.G. (1976) Variability of Skin Temperature in the Waking Monkey. American Journal of Physiology, 230, 449-455.

[16] Minagawa, M., Narita, J., Tada, T., Maruyama, S., Shimizu, T., Bannai, M., Oya, H., Hatakeyama, K. and Abo, T. (1999) Mechanisms Underlying Immunologic States during Pregnancy: Possible Association of the Sympathetic Nervous System. Cellular Immunology, 196, 1-13. http://dx.doi.org/10.1006/cimm.1999.1541

[17] Mori, H., Nishijo, K., Kawamura, H. and Abo, T. (2002) Unique Immunomodulation by Electro-Acupuncture in Humans Possibly via Stimulation of the Autonomic Nervous System. Neuroscience Letters, 320, 21-24. http://dx.doi.org/10.1016/S0304-3940(02)00012-5

[18] Yen, S.S. (1977) Regulation of the Hypothalamic-Pituitary-Ovarian Axis in Women. Journal of Reproduction and Fertility, 51, 181-191. http://dx.doi.org/10.1530/jrf.0.0510181

[19] Adam, D. (2000) No Sweat. Nature. https://www.google.co.jp/webhp?sourceid=chrome-instant\&ion=1\&espv=2\&ie=UTF-8\#q=doi\%3A10.1010\%2Fnews0 00615-4

[20] Sagiyama, K., Tsuchida, M., Kawamura, H., Wang, S., Li, C., Bai, X., Nagura, T., Nozoe, S. and Abo, T. (2004) Age-Related Bias in Function of Natural Killer T Cells and Granulocytes after Stress: Reciprocal Association of Steroid Hormones and Sympathetic Nerves. Clinical \& Experimental Immunology, 135, 56-63. http://dx.doi.org/10.1111/j.1365-2249.2004.02340.x

[21] Brosens, J.J., Salker, M.S., Teklenburg, G., Nautiya, J., Salter, S., Lucas, E.S., Steel, J.H., Christian, M., Chan, Y.W., Boomsma, C.M., Moore, J.D., Hartshorne, G.M., Sućurović, S., Mulac-Jericevic, B., Heijnen, C.J., Quenby, S., Koerkamp, M.J., Holstege, F.C., Shmygol, A. and Macklon, N.S. (2014) Uterine Selection of Human Embryos at Implantation. Scientific Reports, 4, 3894. http://dx.doi.org/10.1038/srep03894 